Review

\title{
Advances in Multi-Omics Study of Prognostic Biomarkers of Diffuse Large B-Cell Lymphoma
}

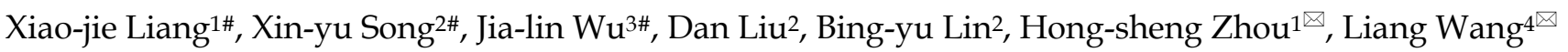 \\ 1. Department of Hematology, Nanfang Hospital, Southern Medical University, Guangzhou 510515, China. \\ 2. The First Clinical Medical College, Guangdong Medical University, Zhanjiang 524000, China. \\ 3. Department of Interventional Radiology, The Third Affiliated Hospital of Sun Yat-sen University, Guangzhou 510630, China. \\ 4. Department of Hematology, Beijing Tongren Hospital, Capital Medical University, Beijing 100730, China. \\ \#The first three authors contributed equally to this work.
}

$\triangle$ Corresponding authors: Liang Wang, MD, Department of Hematology, Beijing Tongren Hospital, Capital Medical University, Beijing 100730, China. Office Phone: +861058266633; Email: wangliangtrhos@126.com; Hong-sheng Zhou, MD, PhD, Department of Hematology, Nanfang Hospital, Southern Medical University, Guangzhou 510515, China, E-mail: hanson_tcm@126.com

(C) The author(s). This is an open access article distributed under the terms of the Creative Commons Attribution License (https://creativecommons.org/licenses/by/4.0/). See http://ivyspring.com/terms for full terms and conditions.

Received: 2021.10.09; Accepted: 2021.12.31; Published: 2022.01.09

\begin{abstract}
As the most common subtype of non-Hodgkin's lymphoma, diffuse large B-cell lymphoma (DLBCL) is characterized by a huge degree of clinical and prognostic heterogeneity. Currently, there is an urgent need for highly specific and sensitive biomarkers to predict the therapeutic response of DLBCL and assess which patients can benefit from systemic chemotherapy to help develop more precise therapeutic regimens for DLBCL. Systems biology (holistic study of diseases) is more comprehensive in quantifying and identifying biomarkers, helps addressing major biological problems, and possesses high accuracy and sensitivity. In this article, we provide an overview of research advances in DLBCL prognostic biomarkers made using the multi-omics approach of genomics, transcriptomics, epigenetics, proteomics, metabonomics, radiomics, and the currently developing single-cell technologies.
\end{abstract}

Key words: Diffuse large B cell lymphoma; Prognosis; Precision medicine; Systems biology; Biomarkers

\section{Introduction}

Diffuse large B cell lymphoma (DLBCL) is the most common malignancy of the lymphohematopoietic system in adults, accounting for approximately $35 \%$ of non-Hodgkin's lymphomas [1] and has an aggressive clinicopathological course [2]. In recent years, there have been significant improvements in the diagnosis and therapy of DLBCL. Its treatment is mainly immunochemotherapy-based. Forty to fifty percent of the DLBCL patients remain incurable after first-line treatment with rituximab, cyclophosphamide, adriamycin, vincristine, and prednisone (R-CHOP) [3]. Therefore, highly specific and sensitive prognostic biomarkers are needed for exploring novel therapeutic targets, developing personalized therapies and posttreatment monitoring of DLBCL patients, ultimately improving their survival rate. Additionally, the heterogeneity and high recurrence rate of DLBCL makes early prognostic biomarkers essential for the development of treatment regimens and prognosis assessment.

Recent studies have shown that there are differences in predicting the prognosis of DLBCL patients based on cell origin classification. In the last decade, the advent of high-throughput genomic sequencing platforms, particularly whole-exome sequencing has helped identifying many genetic alterations unique to DLBCL [4]. Systems biology approach, which allows researchers to observe and solve medical problems from a holistic perspective by combining data from genomics, transcriptomics, epigenetics, proteomics, metabolomics, and imaging, may be better suited to identify prognostic biomarkers for DLBCL. In this review, we have systematically reviewed the various multi-omics researches on DLBCL prognostic biomarkers (Figure 1), aiming to promote the precision medicine in DLBCL. 


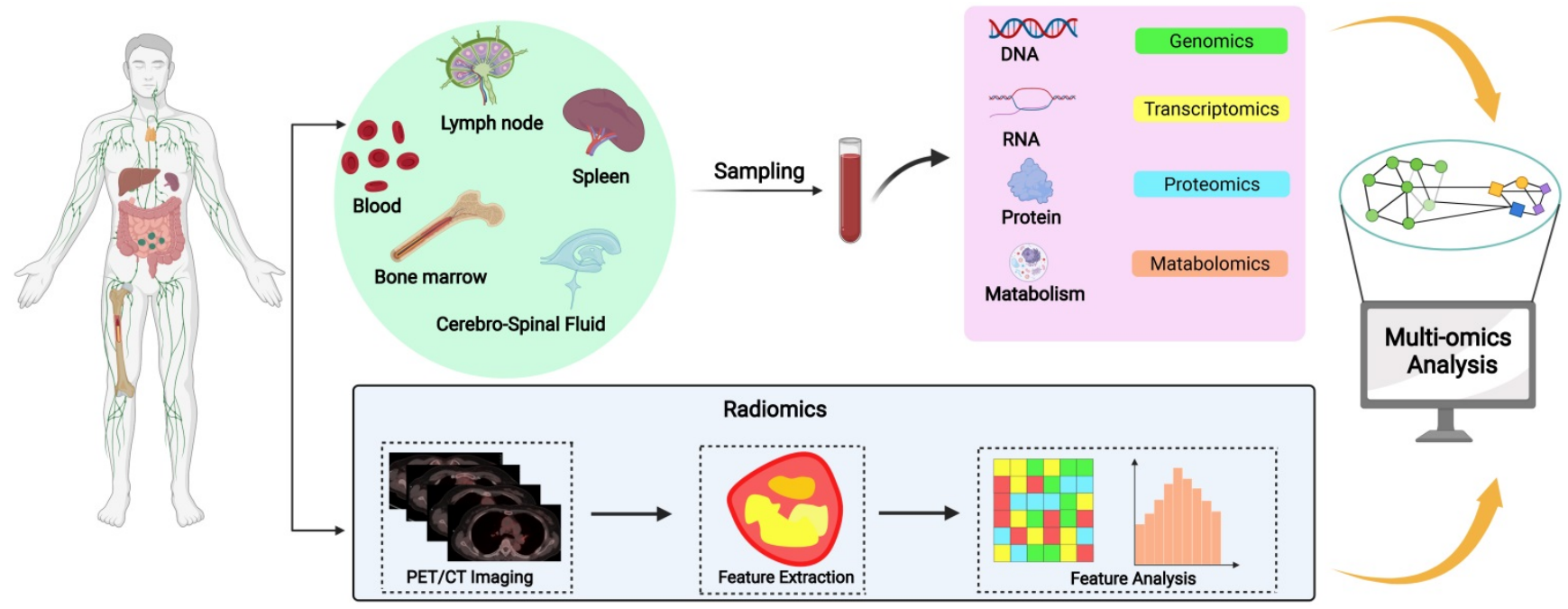

Figure 1: A systematic review of prognostic biomarkers of DLBCL. Diverse sample sources including lymph nodes, spleen, bone marrow, blood and cerebrospinal fluid provide diverse options for the screening of DLBCL biomarkers. Systems biology integrates data from multi-omics studies and uses multiple methods to analyze and process the data to gain a comprehensive understanding of the molecular mechanisms of DLBCL and identify prognostic biomarkers, but these biomarkers must be validated through clinical trials before clinical application.

\section{Biomarkers of DLBCL subtypes}

In 2000, Alizadeh et al. classified DLBCL into two subtypes based on its gene expression profile obtained using DNA microarray: germinal center B cells (GCB) and activated B cells (ABC) [5]. They suggested that the overall survival (OS) of patients with the GCB subtype was significantly longer than that of patients with the ABC subtype. Subsequently, in 2002, Rosenwald et al. used DNA microarrays to determine gene expression in 240 DLBCL biopsy samples and identified three gene expression subgroups of DLBCL: GCB-like, ABC-like, and unclassified DLBCL. Since the survival curves of ABC-like DLBCL and unclassified DLBCL are inseparable, they are now collectively referred as non-germinal center B cell-like (non-GCB) [6]. Although accurate, these molecular subtyping are costly and complicated, making their clinical implementation difficult. Therefore, Hans et al. proposed immunohistochemistry (IHC) as an alternative to microarray testing. They found that the presence of three proteins, CD10, BCL-6, and MUM1, could be used as a basis for DLBCL subtyping. DLBCL subtyping and survival analysis based on its IHC profile did not differ significantly from molecular subtyping [7]. However, about $20 \%$ disconcordance may occur between Hans's algorithm and genomic subtyping [8]. Thus, genomic analysis should be done to confirm the subtyping when it is highly suspected that the clinical findings do not match the Hans subtyping.

Molecular variant landscape of DLBCL has improved with the development of the next-generation sequencing technology (NGS). The increasingly accurate NGS-based molecular subtyping of DLBCL has greatly improved the prognostic assessment of patients. Schmitz et al. carried out exome and transcriptome sequencing on 574 DLBCL biopsy samples. They conducted DNA copy number analysis using chip technology, and targeted amplicon resequencing of 372 genes. Consequently, they identified four prominent genetic subtypes in DLBCL, termed MCD (based on the co-occurrence of MYD88 ${ }^{\mathrm{L} 265 \mathrm{P}}$ and CD79B mutations), BN2 (based on BCL6 fusions and NOTCH2 mutations), N1 (based on NOTCH1 mutations), and EZB (based on EZH2 mutations and BCL2 translocations). This tetrad subtyping system is valuable in predicting outcomes, with BN2 and EZB subtypes having better survival than that of MCD and N1 subtypes [9]. Increasing studies have confirmed the efficacy of BTK inhibitors in MCD subtype, especially patients with primary central nervous system DLBCL, 37\% of whom are classified as MCD subtype [10]. However, $53.4 \%$ of the patients could not be typed using this method. Using data from 304 DLBCL patients, Chapuy et al. performed a multilevel genetic analysis pertaining to low-frequency alterations, captured recurrent mutations, somatic copy number alterations (SCNAs), and structural variants (SVs). They used a consistent clustering method for integration to identify five DLBCL subtypes: 1) Cluster 1 (C1), 2) Cluster 2 (C2), 3) Cluster 3 (C3), 4) Cluster 4 (C4) and, 5) Cluster 5 (C5). The C1 subtype is a previously unidentified group of low-risk ABC-DLBCLs; the C2 subtype often exhibits 9p21.13/CDKN2A and 13q14.2/RB1 copy deletions; $95 \%$ of C3 subtypes are of GCB origin and exhibit BCL2, KMT2D, CREBBP, and EZH2 mutations, PTEN 
inactivation and epigenetic enzyme alterations; $\mathrm{C} 4$ subtypes are a newly defined group of high-risk GCB-DLBCLs; and the C5 subtype exhibits increased $18 \mathrm{q}$ copy number and high BCL2 expression. Although both $\mathrm{C} 4$ and $\mathrm{C} 3$ are predominantly GCB types, unlike the PETN alterations in C3, they are more common in C4 RHOA mutations [1]. In 2020, Stuart et al. analyzed the mutation characteristics of a population-based cohort of 928 DLBCL patients by targeted sequencing of 293 genes and applied Bernoulli mixture-model clustering to classify patients into five molecular subtypes: 1) MYD88, 2) BCL2, 3) SOCS1/SGK1, 4) TET2/SGK1, and 5) NOTCH2, along with a "not elsewhere classified (NEC)" group. The BCL2 subtype, SOCS1/SGK1 subtype, and TET2/SGK1 subtype had good prognosis, with 5-year OS rates of $62.5 \%, 64.9 \%$, and $60.1 \%$, respectively; the MYD88 subtype had the worst prognosis, with a 5-year OS of $42.1 \%$; NOTCH2 subtype and NEC subtype had intermediate prognosis with 5-year OS of $48.1 \%$ and $53.6 \%$, respectively [11].

Wright et al. subtyped tumors based on the genetic profile of DLBCL patients. They created the LymphGen algorithm to provide a probabilistic classification of tumors that translate from individual patients to genetic subtypes, allowing for the classification of DLBCL into seven genetic subtypes: 1) $\mathrm{MCD}$, 2) N1, 3) A53, 4) BN2, 5) ST2, 6) EZB-MYC+, and 7) EZB-MYC-, with a patient prevalence of $8.7 \%$, $1.7 \%, 5.8 \%, 13.3 \%, 6.4 \%, 5.9 \%$, and $17.6 \%$, respectively, and 5 -year OS rates of $40 \%, 27 \%, 63 \%, 67 \%, 84 \%, 48 \%$, and $82 \%$, respectively [12]. This novel genetic subtyping system was successfully applied in guiding personalized treatment for newly diagnosed DLBCL. In the study reported by WL Zhao et al, ibrutinib was added to R-CHOP for MCD and BN2 subtypes, lenalidomide for $\mathrm{N} 1$ and not otherwise specified (NOS) subtypes, decitabine for A53 subtype, tucidinostat for EZB subtype. This genetic subtype-guided R-CHOP plus $\mathrm{X}$ model $(\mathrm{R}-\mathrm{CHOP}+\mathrm{X}$ ) was demonstrated to be superior over the classic $\mathrm{R}-\mathrm{CHOP}$ regimen, and attained both higher $\mathrm{CR}$ rate and longer PFS [13].

Recently, Kotlov et al. reconstructed the lymphoma microenvironment (LME) of 4580 DLBCL patients using 25 functional gene expression signatures (FGES). They used an unsupervised community detection algorithm, which was used to analyze the correlation of FGES among samples, resulting in four major LME clusters: 1) germinal center-like LMS (GC-like LMS), enriched in FGES from cell types commonly found in germinal centers; 2) Mesenchymal subtype (MS-LME, abundant in FGES from stromal cells and extracellular matrix pathways;
3) Inflammatory subtype(IN-LME), enriched in FGES associated with inflammatory cells and pathways; and 4) Depleted subtype(DP-LME, was characterized by an overall lower abundance of microenvironment derived FGES). Microenvironment derived FGES accounted for $15 \%, 33 \%, 25 \%$, and $27 \%$ of the four subtypes, respectively. This LME-based categorization represents a new gene expression-based DLBCL subtyping and is to a degree related to the cell of origin (COO)-based approach. The IN-LME subtype contains a higher proportion of ABC-DLBCLs, but GC-like and MS-LME are more abundant in GCB-DLBCLs. These four different subtypes have different prognoses. Overall, GC-like and MS-LME subtypes confer a better prognosis, with a 5-year survival rate of approximately $80 \%$, while, IN-LME and DP-LME subtypes confer poor prognosis. Especially DP-LME has a 5-year patient survival (PS) rate of approximately $60 \%$ [14]. These researches are displayed in Table 1.

\section{Genomics}

High-grade B cell lymphoma with MYC and BCL2 and/or BCL6 translocations is defined as a new entity in the 2016 WHO classification [15]. Mounting evidences have confirmed that MYC/BCL2 co-expression and double translocations are important determinants of prognosis in DLBCL patients [16]. MYC rearrangements occur in 5\%-10\% of DLBCL [17], half of which also have BCL2 rearrangements [18]. Staiger et al. found that dual expression of MYC and BCL2 suggested a poor prognosis for DLBCL [19]. The gene STAT3 located on human chromosome 17 regulates cell growth by upregulating MYC, a downstream target [20]. Constitutive STAT3 activation is a distinctive feature of ABC subtypes in DLBCL. Huang et al. studied 185 DLBCL patients treated with R-CHOP and using IHC identified phosphotyrosine STAT3 (PY-STAT3) expression. Cell line-based siRNA assays also yielded an 11-gene PY-STAT3 activation signature, indicating that STAT3 activation is associated with poor survival in DLBCL R-CHOP treated with patients, particularly those with the $A B C$ subtype [21].

Meta-analysis of four studies by Ghesquieres et al. revealed that a two-single nucleotide polymorphism (SNP) risk score can highly predict event free survival (EFS) $\left(p=1.78 \times 10^{-12}\right)$ and is independent of treatment, International Prognostic Index (IPI), and cell source classification [22]. They genotyped SNP loci, and used a log-additive genetic model with adjustment for age, sex and an age-adjusted IPI to calculate the hazard ratios (HRs) of EFS and OS in the sample and 95\% confidence interval (CI). They found that the trait locus markers 
of EFS are $5 q 23.2$ rs7712513 (close to SNX2 and SNCAIP genes; HR 1.39, 95\% CI 1.23-1.57; $P=$ $2.08 \times 10^{-7}$ ) and $6 \mathrm{q} 21$ rs7765004 (close to MARCKS and HDAC2 genes; HR 1.38, 95\% CI 1.22 - 1.57; $P$ $\left.=7.09 \times 10^{-7}\right)$; the loci rs7712513 (HR 1.49, 95\% CI 1.29 1.72; $\left.P=3.53 \times 10^{-8}\right)$ and rs7765004 (HR 1.47, 95\% CI $1.27-1.71 ; P=5.36 \times 10^{-7}$ ) were related to the patient's OS.

Using NGS technology, Pasqualucci et al. sequenced the whole-exome of 115 DLBCL samples and found that presence of multiple lesions targeting histone/chromatin modifying genes is a significant feature of the DLBCL tumor cell genome. Among them, mixed-lineage leukemia 2 (MLL2) is the most common mutation. In addition, they also found that $\beta 2$-microglobulin (B2M) gene also has frequent mutations and deletions. Because the $\mathrm{B} 2 \mathrm{M}$ gene is related to $\mathrm{T}$ cell immune recognition, its high frequency mutation or deletion makes DLBCL tumor cells insensitive to cytotoxic T cell-mediated killing, suggesting that tumors evade immune surveillance mainly via B2M deletion [23]. Thus, B2M deletion may be a new prognostic indicator in DLBCL patients. Whole-exome sequencing and RNA sequencing
(RNA-seq) of samples from 1,001 newly diagnosed DLBCL patients treated with rituximab-containing regimens, by Reddy et al., revealed 150 genetic drivers. Key oncogenes related to tumor cell growth (MYC, RHOA, SF3B1, MTOR, and BCL2) and genes related to tumor suppression (TP53, MGA, PTEN, and NCOR1) were identified. Knockout studies of the nine identified functional oncogenes showed that knockout of EBF1, IRF4, CARD11, MYD88, and IKBKB is selectively lethal in ABC DLBCL, while knockout ZBTB7A, XPO1, TGFBR2, and PTPN6 is selectively lethal in GCB DLBCL. Thus, they can be used as targets for the treatment of DLBCL patients and as biomarkers for predicting patient treatment outcomes. More importantly, $36 \%$ of DLBCL patients have genetic mutations in these nine drug targets and may thus respond well to chemotherapy. In addition, they also developed a genomic risk model and found that the 5-year survival rates of high-risk and low-risk patients in the complete remission group were approximately $60 \%$ and $90 \%$, respectively, while the IPI was approximately $50 \%$ and $85 \%$, respectively, indicating that the genomic risk model can make early prognostic predictions in DLBCL patients [24].

Table 1. Summary description of DLBCL subtyping and main research methods.

\begin{tabular}{|c|c|c|c|c|}
\hline Year & Journal & Author & Methods & Subtype \\
\hline 2000 & Nature & Alizadeh et al [5] & $\begin{array}{l}\text { DNA microarray analysis of gene } \\
\text { expression }\end{array}$ & $\begin{array}{l}\text { - GCB: GC B-like DLBCL } \\
\text { - ABC: activated B-like DLBCL }\end{array}$ \\
\hline 2002 & N Engl J Med & Rosenwald et al [6] & $\begin{array}{l}\text { DNA microarrays and analyzed for } \\
\text { genomic abnormalities }\end{array}$ & $\begin{array}{l}\text { - GCB: germinal-center B-cell-like } \\
\text { - ABC: activated B-cell-like } \\
\text { - unclassified DLBCL: type } 3 \text { diffuse large-B-cell lymphoma }\end{array}$ \\
\hline 2004 & Blood & Hans et al [7] & Immunohistochemistry & $\begin{array}{l}- \text { GCB: } \\
\text { 1)CD10(+) } \\
\text { 2)bcl-6(+)\& CD10+(+) } \\
\text { 3)bcl-6(+),CD10(-)\& MUM1(-) } \\
\text { non-GCB: } \\
\text { 1)bcl-6(-)\& CD10(+) } \\
\text { 2)bcl-6(+),CD10(-)\& MUM1(+) }\end{array}$ \\
\hline 2018 & N Engl J Med & Schmitz et al [8] & $\begin{array}{l}\text { Exome and transcriptome sequencing, } \\
\text { array-based DNA copy-number analysis, } \\
\text { and targeted amplicon resequencing }\end{array}$ & $\begin{array}{l}\cdot \text { MCD (co-occurrence of MYD88L265P and CD79B mutations) } \\
\cdot \text { BN2 (BCL6 fusions and NOTCH2 mutations) } \\
\cdot \text { N1 (NOTCH1 mutations) } \\
\cdot \text { EZB (EZH2 mutations and BCL2 translocations) }\end{array}$ \\
\hline 2018 & Nat Med & Chapuy et al [1] & Comprehensive genetic analysis & $\begin{array}{l}\text { - Cluster } 1 \text { (BCL6 SVs, mutations of NOTCH2 signaling pathway ) } \\
\text { - Cluster } 2 \text { (biallelic inactivation of TP53, copy loss of 9p21.13/CDKN2A) } \\
\text { - Cluster } 3 \text { (BCL2 SVs and alterations of PTEN and epigenetic enzymes) } \\
\text { - Cluster } 4 \text { (distinct alterations in BCR/PI3K, JAK/STA T and BRAF pathway) } \\
\text { - Cluster } 5 \text { (18q gain, frequent mutations in CD79B and MYD88.) }\end{array}$ \\
\hline 2020 & Blood & Stuart et al [9] & Targeted sequencing & $\begin{array}{l}\text { - MYD88: Strongly associated with ABC-type DLBCL, a poor prognosis } \\
\text { - BCL2: Strongly associated with GCB-type DLBCL, generally favorable prognosis } \\
\text { - SOCS1/SGK1: Predominantly GCB-type DLBCL, the most favorable prognosis } \\
\text { - TET2/SGK1: A less strongly identifiable subtype, a favorable prognosis } \\
\text { - NOTCH2: Not associated with any cell of origin, intermediate survival } \\
\text { - NEC: A default category, intermediate survival }\end{array}$ \\
\hline 2020 & Cancer Cell & Wright et al [10] & LymphGen algorithm & $\begin{array}{l}\text { MCD: } 5 \text {-year OS rate of } 40 \% \\
\text { - N1: 5-year OS rate of } 27 \% \\
\text { A53: 5-year OS rate of } 63 \% \\
\text { - BN2: } 5 \text {-year OS rate of } 67 \% \\
\text {-ST2: } 5 \text {-year OS rate of } 84 \% \\
\text { - EZB-MYC+: } 5 \text {-year OS rate of } 48 \% \\
\text { - EZB-MYC-: } 5 \text {-year OS rate of } 82 \%\end{array}$ \\
\hline 2021 & Cancer Discov & Kotlov et al [11] & $\begin{array}{l}\text { Transcriptomic analysis of the } \\
\text { microenvironment }\end{array}$ & $\begin{array}{l}\text { - GC-like-LME: commonly found in germinal centers } \\
\text { - MS-LME: abundant in stromal cells and extracellular matrix pathways } \\
\text { - IN-LME: associated with inflammatory cells and pathways } \\
\text { - DP-LME: an overall lower presence of microenvironment-derived FGES }\end{array}$ \\
\hline
\end{tabular}


Circulating tumor DNA (ctDNA), DNA fragments released into the circulatory system by cancer cells, contain tumor-specific information. They are independent biomarkers used to assess the prognosis of DLBCL patients. ctDNA can be detected and quantified using NGS technology and allows noninvasive assessment of tumor kinetics [25, 26]. Studies indicate a strong association between ctDNA and total metabolic tumor volume (TMTV) in DLBCL patients [27]. Sidaway et al. studied 217 patients with DLBCL or primary mediastinal large B-cell lymphoma and found that ctDNA was measurable in $98 \%$ of the patients prior to treatment. These patients were subsequently divided into the discovery $(n=130)$ and validation $(n=73)$ groups. The dynamics of their ctDNA levels were monitored throughout treatment. ctDNA levels in responders reduced significantly within the first week of treatment. Thus, responders and non-responders could be identified at the end of the first treatment cycle. This finding was validated in the exploratory group: patients with early molecular response (EMR) and major molecular response (MMR), the 24-month EFS rate increased significantly, $83 \%$ vs. $50 \%(P=0.0015)$ and $82 \%$ vs. $46 \%(P<0.001)$, and found that both EMR and MMR can predict the prognosis of DLBCL patients independently of the imaging results of positron emission computed tomography (PET-CT). These data indicate that the dynamic changes in ctDNA level can be early predictors of clinical prognosis in DLBCL patients, and ctDNA-based prognostic predictions do not depend on PET-CT imaging [28]. In 2015, Roschewski et al. performed ctDNA quantitative analysis in a cohort of newly diagnosed DLBCL patients by monitoring serum ctDNA in a series of samples obtained during treatment and follow-up. They found that the rate of 5-year time to disease progression of patients with and without metaphase ctDNA was $41.7 \%$ (95\% CI $22.2 \%-60.1 \%$ ) and $80.2 \%$ (95\% CI $69.6 \%-87.3 \%$ ), respectively, and the OS rate was $65.4 \%$ (95\% CI $42.4 \%-81.1 \%$ ) and $83 \%$ (95\% CI $73.1 \%-89.6 \%$ ); the positive predictive value (PPV) of ctDNA was $63 \%$ and the negative predictive value (NPV) was $80 \%$, with sensitivities and specificities of $47 \%$ and $88 \%$, respectively. Therefore, ctDNA is better than PET-CT for predicting outcome and treatment response in DLBCL patients, suggesting that ctDNA may be used as predictive prognostic biomarkers in PET-CT-negative patients [29].

\section{Transcriptomics}

Transcriptomic studies typically use RNA sequencing (RNA-seq), real-time quantitative PCR (qPCR), or microarray technology. A common application of transcriptomics in DLBCL research is to search for transcripts with altered expression in normal B lymphocytes, tumor cells, and different subtypes of tumor cells. In addition, non-coding RNAs, including microRNAs (miRNAs) and long non-coding RNAs (lncRNAs), also play important regulatory functions. Importantly, transcriptomic studies allow quantitative assessment of transcripts at various time points throughout the course of treatment, this information is essential for the prognostic assessment of DLBCL.

The biological basis of high-grade DLBCL with MYC, BCL2, and/or BCL6 rearrangements (HGBL-DH/TH-BCL2) was explored by Ennishi et al. They analyzed whole-exome sequencing, RNA-seq, and targeted resequencing and other data from patients with GCB-DLBCL $(\mathrm{n}=157)$, including cases of HGBL-DH/TH-BCL2 ( $\mathrm{n}=25)$. They constructed a 104-gene (mRNA) double-hit signature (DHITsig) model for estimating OS rate using Kaplan-Meier survival analysis and found that the 5-year OS rate after R-CHOP treatment in DHITsig-negative (DHITsig-neg) and DHITsig-positive (DHITsig-pos) patients was $80 \%$ and $60 \%$, respectively. Thus, they demonstrated the predictive utility of DHITsig on the prognostic outcome of DLBCL patients [30].

Recently, tumor microenvironment (TME) has been found to be critical in mediating immune evasion and treatment resistance in various cancers. Ciavarella et al. used the CIBERSORT algorithm to deconvolute gene expression profile (GEP) data from 482 untreated DLBCL patients and identified 45 tumor microenvironment (TME) genes of DLBCL. They quantified the expression of 45 tumor microenvironment (TME) genes in formalin-fixed, paraffin-embedded biopsy tissue samples using NanoString technique, and found that the 30-month OS rate was approximately $90 \%$ and $62.5 \%$ for those with high and low expression of all genes in TME, respectively $(P=0.00017)$, and progression free survival (PFS) rate was approximately $75 \%$ and $60 \%$, respectively $(P=0.0069)$; COO/TME combination model predicted a 30 -month OS rate of $<25 \%$ and $>75 \%$ in the high-risk and low-risk groups, respectively $(P=0.00022)$. Thus, they concluded that TME has good prognostic risk stratification ability and its predictive power is significantly improved when combined with $\mathrm{COO}$ [31].

miRNAs can be used as prognostic biomarker of DLBCL. Sun et al. used miRNA PCR arrays to analyze the miRNA expression profiles DLBCL patients at three time points of treatment (372 serum samples from 20 patients), viz. diagnosis, remission, and relapse. They identified and used four miRNAs, i.e., miR21, miR130b, miR155, and miR28 to establish a 
4-loop miRNA prognostic model. Multivariate analysis showed that the 4-loop miRNA prognostic model was significantly associated with poorer PFS and OS in both the training and validation cohorts [32]. Unlike some investigators who focused mainly on collecting pre-treatment specimens, Bouvy et al. collected 68 miRNA samples from the sera of 19 DLBCL patients during treatment and analyzed them using microarray and qPCR techniques. They found that the plasma levels of miR-197, miR-20a, miR-451, miR-122, miR-19b, and miR-21 were closely associated with the sensitivity of DLBCL patients to chemotherapy, reflecting their prognostic utility in DLBCL patients. However, this preliminary study needs to be validated using a larger sample size [33].

In addition to miRNAs, IncRNAs have also shown important prognostic assessment value. Zhuo et al. studied three cohorts, including GSE31312 ( $\mathrm{n}=$ 426), GSE10846 $(n=350)$, and GSE4475 $(n=129)$ from the Gene Expression Omnibus (GEO) database. Using differential expression analyses and weighted voting algorithm, they found that SubSigLnc-17, an lncRNA recognition marker is capable of distinguishing between GCB and ABC subtypes, with a sensitivity of 92.5\%. SubSigLnc-17 was also identified for prognostic prediction [34]. Although non-coding RNAs have predictive significance for DLBCL prognosis, the clinical application of miRNAs or IncRNAs as prognostic biomarkers for DLBCL is still relatively rare.

The main challenge in identifying biomarkers with transcriptomics, is the requirement of large number sample size, making it expensive. In future, the biomarker study will continue to be influenced by the rapid developments in transcriptomics albeit with newer unknown challenges. In addition, transcriptomics is not useful in cases where the disease is not caused by alterations in RNA sequences. Proteomics may be suited to study biomarkers in such cases.

\section{Proteomics}

Proteomics encompasses identification of proteins and their posttranslational modifications, functions, and protein-protein interactions using two-dimensional chromatography (2D-LC) and two-dimensional liquid chromatography/tandem mass spectrometry (LC-MS/MS). Clinically, proteins or peptides expressed in tumor cells and body fluids of DLBCL patients have the potential to be prognostic DLBCL biomarkers. Recent developments in proteomics techniques, including increased sensitivity of detection has helped immensely in the discovery of novel prognostic biomarkers.

Some DLBCL tumor cells express programmed death receptor-ligand 1 (PD-L1) and its receptor the programmed death receptor 1 (PD-1) is expressed on $\mathrm{T}$ lymphocytes surface. The PD-1/PD-L1 pathway mediates immune escape from tumors. Therapies targeting the PD-1/PD-L1 pathway are clinically effective in treating Non-Hodgkin's Lymphoma (NHL) $[35,36]$. Kiyasu et al. suggested that while positive surface-expression of PD-L1 in DLBCL tumor cells is an independent prognostic factor for OS, the expression of PD-L1 in microenvironmental cells is not correlated with OS [37]. DLBCL cells aberrantly express oncogenic transcription factor forkhead box protein 1 (FOXP1). Flori et al. found that DLBCL cells with aberrantly high FOXP1 expression had reduced expression of surface sphingosine-1-phosphate receptor 2 (S1PR2) [38]. Since S1PR2 is required for the maintenance of GCB cell homeostasis [39], using an additional publicly available 2-gene expression profile (GEP) dataset they found that S1PR2 showed a near perfect negative correlation with FOXP1. Thus, suggesting that low S1PR2 expression, especially in combination with high FOXP1 expression, is an important predictor of poor prognosis in DLBCL patients [38]. Certain leukocyte antigens (CD) expressed on B-lymphocyte membranes can be used as prognostic markers in DLBCL. Niitsu et al. in a cohort of 930 DLBCL patients found that the 5-year OS rate was $55 \%$ and $65 \%$ for CD5+ DLBCL $(n=102)$ and CD5- DLBCL $(\mathrm{n}=828)$, respectively, while the 5 -year PFS rates were $52 \%$ and $61 \%$, respectively. The addition of rituximab to chemotherapy in patients with CD5+ DLBCL revealed a significant improvement in PFS (47.4\% vs. $62.5 \%)$ at 4 years, but not in OS (57.8\% vs. $63.5 \%)$ at 4 years [40]. Therefore, they concluded that CD5+ could be a predictive biomarker of poor prognosis and response to rituximab therapy in DLBCL. Xu-Monette et al., found that CD37- DLBCL had significantly worse OS and PFS and significantly lower survival rate compared to CD37+ DLBCL patients after R-CHOP treatment. Thus suggesting that $\mathrm{CD} 37+$ is predictive of a benign prognosis in DLBCL patients [41]. Meriranta et al. found that low Kelch-like protein 6 (KLHL6) expression predicted poor prognosis in DLBCL patients [42].

Maurer et al. using the FREELITE assay [43] found that $32 \%$ and $14 \%$ of patients (total $n=219$ ) had elevated pretreatment serum free light chain (sFLC) or $\kappa: \lambda$ FLC abnormalities; DLBCL patients with elevated sFLC had poorer EFS and OS compared to patients with normal sFLC. Therefore, they hypothesized that elevated sFLC was a stronger predictor of poor prognosis in patients with DLBCL [44]. Witzig et al. conducted a 6-year follow-up study monitoring FLC concentrations and found that DLBCL patients fall 
into FLC monoclonal and polyclonal groups $(\mathrm{n}=276$ untreated) (monoclonal group EFS: HR 3.56, 95\% CI 1.88-6.76, $P<0.0001$; polyclonal group EFS: HR 2.56, $95 \%$ CI 1.50-4.38, $P=0.0006$ ), suggesting that elevated FLC is a poor prognostic factor in DLBCL patients and may provide a new target for the treatment of DLBCL patients [45].

Proteomics has enabled a deeper understanding of the aberrant molecular alterations in DLBCL and has the potential to identify new prognostic markers and establish new molecular typing methods. Data on serum FLC in DLBCL patients are limited. Large sample size studies need to be performed and validated to enable faster clinical application. However, there are still some limitations and challenges with proteomics. First, large size of proteomics data makes their storage and processing into biologically meaningful results tough. Second, structural aberrations in protein and very low abundance may cause protein signals to be missed especially when using mass spectrometry (MS).

\section{Epigenetics}

Epigenetics refers to study of heritable changes in gene expression such as histone modifications, DNA methylation, chromatin accessibility and long-range chromatin interactions [46], and chemical modifications of DNA and histones and the conformation of DNA [47] in nucleus determine epigenetics [48].

DNA methylation regulates gene expression, developmental processes and diseases [49]. Intra-tumor methylation heterogeneity in DLBCL patients can predict the time to recurrence [50]. Cytidine deaminase (AICDA) promotes B-cell demethylation in germinal centers. Teater et al. suggested that AICDA overexpression may be a biomarker of poorer prognostic outcomes in DLBCL patients. They found that DLBCL with high AICDA expression had higher intra-tumor methylation heterogeneity and cytosine methylation heterogeneity in tumor cells. The increased cytosine methylation heterogeneity was also associated with poor clinical prognosis in DLBCL patients [51].

N6-methyladenosine (m6A) is the most abundant and prevalent internal co-transcriptional modification in eukaryotic mRNAs. m6A methylation modification plays an important role in cancer development [52]. Han et al. explored the function of m6A methylation modification in DLBCL and found that the high expression of m6A RNA regulatory gene PIWI-interacting RNA 30473 (piRNA) increased the level of $\mathrm{m} 6 \mathrm{~A}$, resulting in poor prognosis in DLBCL patients. Thus, m6A methylation may be used for prognostic stratification in DLBCL [53].
Loss of CD20 is a major obstacle to R-CHOP treatment of relapsed/refractory DLBCL; histone deacetylation in DLBCL patients inhibits CD20 expression. Guan et al. demonstrated for the first time that in vivo and in vitro inhibition of histone deacetylase by chidamide significantly strengthened the tumor inhibitory effect of rituximab leading to improved prognosis in DLBCL patients. Thus, they established a synergistic role for Chidamide in the rituximab-R-CHOP treatment of DLBCL [54]. Thus, it can be noted that histone deacetylation is one of the indicators of poor prognosis in DLBCL patients.

\section{Metabonomics}

The metabolome includes all the low molecular weight (50-1500 Da) metabolites of an organism or cell. Metabonomics is a new discipline of qualitative and quantitative analysis of the metabolome to determine the relative relationship between metabolites and pathophysiological changes in diseases. In recent years, the rapid development of metabolomics has led to its increasing use in the analysis of altered metabolites in DLBCL tumor cells, and can be combined with other histological techniques to better identify prognostic biomarkers in DLBCL patients.

Using tumor cell gene expression as basis and consensus clustering Monti et al. identified three biological isoforms of DLBCL: 1) oxidative phosphorylation (Oxphos), 2) B-cell receptor/ proliferation, and 3) host response (HR) [55]. Oxphos -DLBCL have a predominantly glycolytic energy metabolism [56] enriched for genes involved in oxidative phosphorylation, mitochondrial function and electron transport chain. Patients with the 3 subtypes had similar 5-year survival rates (OxPhos, $53 \%$; BCR/proliferation, $60 \%$; and HR 54\%; $P=0.53$ ) [55] suggesting that there may be metabolic heterogeneity in DLBCL and that this heterogeneity is genome-related. Ceriani et al. examined 103 DLBCL patients with primary mediastinal (thymic) large B-cell lymphoma (PMBCL) using (18) F-fluorodeoxyglucose(18FDG) positron emission tomography/computed tomography (PET/CT). They measured baseline 18F-FDG PET/CT by maximum standardized uptake value (SUVmax), metabolic tumor volume (MTV), and total lesion glycolytic (TLG) defined metabolic activity. All patients received combination chemotherapy based on adriamycin and rituximab; 93 received consolidation radiotherapy with a median follow-up of 36 months, at which time the OS rate was $100 \%$ in patients with low TLG and $80 \%$ in patients with high TLG $(P=0.0001)$, while PFS was $99 \%$ and $64 \%$, respectively $(P<0.0001)$, leading to speculation that elevated TLG may be significantly 
associated with poorer PFS and OS in PMBCL patients, proposing that TLG on baseline PET appears to be a strong predictor of PMBCL outcome [57].

A common metabolic change found in tumor cells is elevated levels of glycolysis [58], and glyceraldehyde-3-phosphate dehydrogenase (GAPDH) is a key enzyme involved in glycolysis. Through unbiased analysis, Chiche et al. determined that GAPDH is the only glycolytic enzyme that can predict OS in DLBCL patients treated with R-CHOP. Based on GAPDH automated immunohistochemical (IHC) staining, a GAPDH scoring system was established. They quantified GAPDH expression levels in paraffinembedded tissue microarray (TMA) of 43 newly diagnosed DLBCL patients and found that high GAPDH expression remained an important marker for predicting improved OS. Multivariate analysis (HR 0.603, $P=0.0371$ ) showed that high GAPDH expression was an independent predictive biomarker for good prognosis in DLBCL patients [59]. Alpha-ketoglutarate (a-KG) is a key metabolite in energy generation via the tricarboxylic acid cycle (TCA cycle) and can be generated during the catabolism of glutamine (Gln) to glutamate (Glu), a reaction catalyzed by aspartate transaminase (GOT1/2). By comparing the expression of GOT2 in normal B cells and different B-cell lymphomas, Feist et al. determined that abnormal GOT2 expression was characteristic of a subgroup of DLBCL. They then studied DLBCL patients $(\mathrm{n}=157)$ treated with $\mathrm{R}-\mathrm{CHOP}$ by including GOT2 expression in a Cox proportional risk model and found high GOT2 expression (HR 2.28, $P=0.03756$ ) was significantly associated with shorter OS in DLBCL patients and could be used as a marker of poor prognosis in DLBCL [60].

Tome et al. (2005) determined that a redox signature score predicted poor prognosis in DLBCL patients, which incorporated features such as vitamin-D3 upregulated protein 1(VDUP1/TIP/ TBP2), MnSOD, ZnSOD, EcSOD, thioredoxin reductase 1 and 2, catalase, thioredoxin, and microsomal GST. Patients with DLBCL were divided into four groups according to the quartiles of the oxidation reduction characteristics score, and their 5-year survival rates were compared: patients in quartiles 1 and 2 had similar 5-year survival rates $(57 \%)$, patients in quartile 3 had lower survival rates $(47 \%)$, and patients in quartile 4 had even lower survival rates $(37 \%)$. Patients in quartile 4 had significantly shorter survival than those in quartile 1 $(P<0.001)$ and quartile $2(P=0.005)$. Thus, they hypothesized that the redox environment may play a role in the prognosis of patients with DLBCL [61]. Kobayashi et al. studied natural killer cells (NK cells) and found that increased lipid metabolism in DLBCL patients produced fatty acids that effectively inhibited NK cell effects and cellular metabolism [62].

Metabolomics makes up for the shortcomings of genomics and proteomics. Its advantages are mainly reflected in the following aspects: A. even tiny genetic mutations have obvious metabolite changes; $B$. the metabolites are fewer in number than genes and proteins, and the study is more comprehensive; C. metabolite changes can directly reflect the pathological state of the organism. With the increasing use of metabolomics in clinical practice and the large number of samples being tested to build up a metabolic profile library, we believe that the study of metabolomic changes in DLBCL patients will become an effective method for identifying prognostic biomarkers in the future.

\section{Single-cell technology}

\section{Single-cell sequencing}

Single-cell sequencing is a new technique of analyzing genome, transcriptome, proteome, and epigenome at the single-cell level using high-throughput sequencing methods [63].

Tumors are composed of multiple cell types, including malignant cells, immune cells, and stromal cell subpopulations. The approach of using bulk tissue multi-omics to holistically understand tumors tends to obscure heterogeneity inherent in tumor cells [64]. In contrast to conventional bulk sequencing, single-cell sequencing can accurately identify heterogeneous cell populations in tumors [65], especially in highly heterogeneous malignancies such as DLBCL.

\section{Single-cell transcriptomics}

Over the past decade, with the development of NGS technology, single-cell RNA sequencing (scRNA-seq) has gradually become an important tool for studying differential gene expression within tumor cells at the transcriptome level [66]. Autologous chimeric antigen receptor (CAR) T-cell therapy targeting CD19 shows good efficacy in patients with DLBCL. Deng et al. infused autologous axicabtagene ciloleucel (axi-cel) anti-CD19 CAR T-cell product in 24 patients with LBCL (2 PMBCL, 6 transformed follicular lymphoma (TFL), and 16 DLBCL), and then selected 137,326 residual cells for the whole transcriptome scRNA-seq test. They carried out a 3 month follow up study on these patients using PET/CT and found that after treatment, $50 \%$ patients experienced progressive disease (PD), $4 \%$ had partial remission (PR), and 38\% had complete remission (CR). By analyzing the cell types in the infusion products of CR patients and PR/PD patients, they 
found a significant enrichment of depleted CD8 and CD4 $\mathrm{T}$ cells in the infusion products of $\mathrm{PR} / \mathrm{PD}$ patients and a significant enrichment of memory CD8 T-cells and three times higher in the infusion products of CR patients than in PR/PD patients. Therefore, they analyzed the differentially expressed genes of CD8 T cells in CR patients and PR/PD patients and found that genes for basic leucine zipper ATF-like transcription factor (BATF), DNA binding inhibitor 2 (ID2), interferon gamma (IFNY), effector molecules (GZMA, GZMB, and GNLY), and major histocompatibility class II (MHCII) molecules were associated with CD8 $\mathrm{T}$ cell depletion. They could serve as genetic markers of CD8 T-cell failure and could also predict the poor prognosis of patients with LBCL under this treatment. In addition, the investigators also measured the fold change of ctDNA alleles relative to somatic mutations in the plasma of patients on day 7 of treatment as early molecular response (EMR) and found that it was significantly correlated with clinical response $(P=0.008)$. Therefore, they suggested that the molecular response on day 7 after CAR T-cell therapy in DLBCL patients may serve as an early predictor of CAR T-cell efficacy markers [67]. Their study provides a novel perspective in the search for molecular biomarkers in patients with LBCL undergoing cellular therapy.

ScRNA-seq emphasizes the importance of intercellular heterogeneity in health and disease phenotypic variability. It has been applied to discover new cell types, explore dynamic developmental processes, identify gene regulatory mechanisms, and reveal random allele expression. Efforts are being made to explore new transcriptome contents, such as spatial transcriptome with the combination of system function analysis and scRNA-seq; however, there is a critical limitation: scRNA-seq can only analyze the RNA profile of each cell once, and the RNA capture efficiency is not completely stable $[68,69]$. Therefore, improving scRNA-seq technology is crucial for the development of single-cell transcriptomes. Currently, single-cell transcriptomics are not well studied in DLBCL. Although there are many challenges, we believe that single-cell transcriptomics will play an important role in the search for prognostic markers in DLBCL.

\section{Other single-cell technologies}

Despite the rapid development of single-cell genomic, single-cell epigenomic, and single-cell proteomic technologies [47, 70-82], their application in DLBCL has not progressed much. Obtaining high-quality and high-coverage genetic data of DNA molecules in single cells, protein concentrations with less noise (with slower protein degradation than
RNA), as well as analyzing various single-cell histological data, remain major challenges that should be overcome [68, 69, 76, 83-85].

Single-cell genomics reveals genomic variability among single cells, transcriptomics, epigenomics and proteomics analyses study the functional state of single cells in an unbiased manner [86]. Precision oncology has rapidly evolved, and proteogenomics is emerging as a new discipline in the clinic and is an important tool for achieving precision oncology therapy. Abundance of mRNA in the cell does not accurately reflect protein abundance, thus combining proteomics and genomics will be more useful for understanding the biological heterogeneity of cancer and identifying different prognostic outcomes in DLBCL patients [87].

\section{Radiomics}

The concept of radiomics was first introduced by Dutch scholar Kumar in 2012 and has become a popular method of image analysis in recent years. Radiomics refers to the use of images obtained using computed tomography (CT), positron emission tomography (PET), or magnetic resonance imaging (MRI) data to extract representative features. Artificial intelligence methods such as machine learning or deep learning are used for quantitative analysis of these images and prediction of diseases. PET is one of the most commonly used imaging technologies for DLBCL diagnosis and treatment processes. The fusion of PET with CT or MRI can provide both functional metabolic and anatomical structural information of tumors, which has an important role in the diagnosis, clinical staging, efficacy prediction, and prognosis assessment of DLBCL.

At the 12th International Conference on Malignant Lymphoma, 2-Fluorine-18-Fluoro-2-deoxyD-glucose-positron emission tomography/ computerized tomography (18FDG-PET/CT) was formally included in the updated guidelines for lymphoma imaging for staging and prognostic assessment [88, 89]. PET/CT is more accurate for DLBCL staging than $\mathrm{CT}$ alone, with upregulated staging being more common than downregulated staging; being highly sensitive to nodal and extra-nodal lesions, PET/CT can accurately assess the sites of these lesions (including skeletal involvement). Now, PET/CT has become a routine part of DLBCL staging and quantifying prognosis. The standard uptake value (SUV) is an indicator used to measure the tumor's ability to take up ${ }^{18}$ FDG. The maximum standard uptake value (SUVmax) of PET/CT at diagnosis was significantly associated with poor survival. 
Interim PET/CT (iPET/CT) is an important auxiliary method for evaluating the efficacy and prognosis of DLBCL. The Deauville score and $\triangle$ SUVmax are two frequently used evaluation methods. Recently, in an iPET/CT evaluation study combined use of Deauville score and $\triangle$ SUVmax was proposed as an improved Deauville model for predicting the DLBCL response to chemotherapy [90]. They retrospectively analyzed 593 patients who received R-CHOP treatment. They found that the 3-year PFS and OS rate of negative patients were $80.2 \%$ and $89.9 \%$, respectively, with a complete response rate of $91.8 \%$, while the 3-year PFS and OS rate of positive patients were only $12.5 \%$ and $27.3 \%$, with a complete response rate of $29.2 \%$, when evaluated by the improved Deauville method. Therefore, they concluded that the method could lead to early identification of chemotherapy-resistant patients, the timely adjustment of the treatment regimen, provision of alternative treatment, and prognostic improvements. However, iPET/CT prognostic assessment has not been standardized yet; further prospective studies are needed [91, 92].

Recent RICOVER-NORT cohort study showed that tumor volume $\geq 7.5 \mathrm{~cm}$ was a poor prognostic factor in elderly patients with aggressive lymphoma [93]. In addition, Laetitia et al. found that total metabolic tumor volume (TMTV) measured at PET/CT baseline was a strong predictor of survival outcomes in patients with DLBCL. In patients treated with lenalidomide maintenance or placebo, elevated TMTV at baseline was significantly associated with poor PFS and OS. They concluded that baseline TMTV combined with parameters that respond to tumor load distribution could improve risk stratification at staging in patients with DLBCL [94].

Lue et al. performed pre-treatment ${ }^{18} \mathrm{FDG}$ PET/CT examinations on 83 patients pathologically diagnosed with DLBCL. They segmented ${ }^{18}$ FDG-avid lesions on PET images using the region-growing algorithm, and standardized lesions with a standardized uptake value (SUV) threshold higher than 2.5 were used for target delineation. Baseline ${ }^{18}$ FDG PET imaging omics feature, RLN GLRLM, was an independent prognostic factor. DLBLC tumors are more aggressive in patients with high RLN $_{\text {GLRLM }}$ as compared to those with low $\mathrm{RLN}_{\mathrm{GLRLM}}$; they have a greater risk of recurrence/progression, and have a lower survival rate. The 5-year PFS and OS rate of patients with high RLN $\mathrm{RLRLM}_{\text {were }} 37.2 \%$ and $41.1 \%$, respectively, while the 5-year PFS and OS of patients

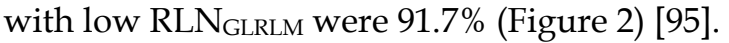

\section{Sampling sources in prognostic biomarker research of DLBCL}

DLBLC biopsy samples can be sourced from various tissues and organs rich in prognostic biomarkers which include lymph nodes, spleen, bone

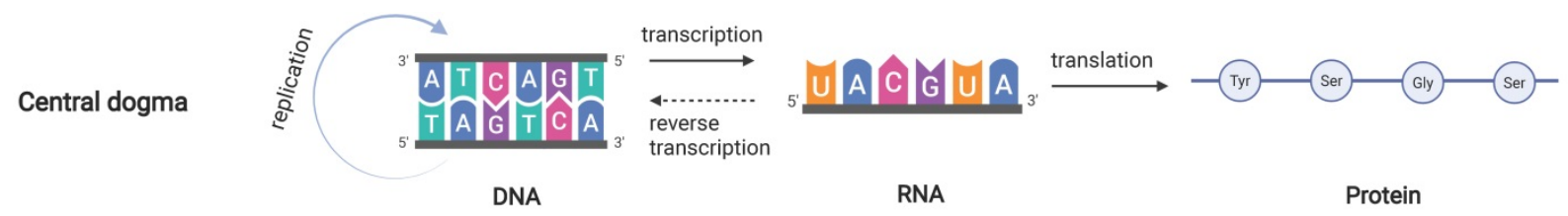

Omics
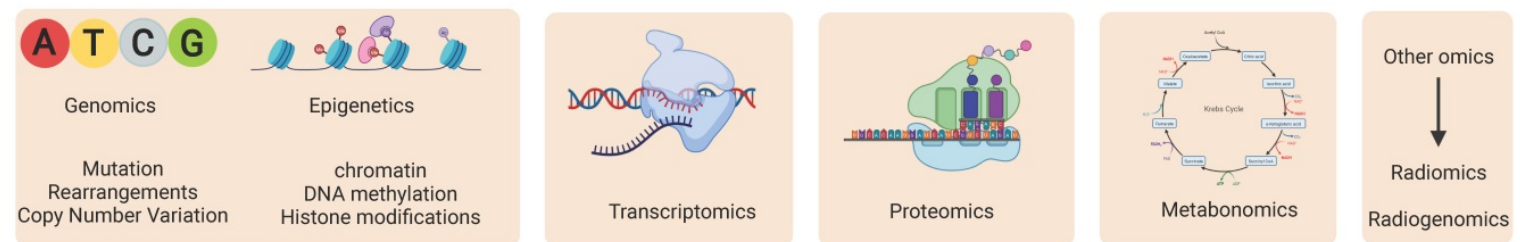

Single cell multi-omics

\begin{tabular}{|c|c|c|c|c|c|c|c|}
\hline \multicolumn{2}{|c|}{ Single-cell sequencing } & \multicolumn{2}{|l|}{ Single-cell genomics } & \multicolumn{2}{|c|}{ Single-cell transcriptomics } & \multicolumn{2}{|c|}{ Single-cell proteomics } \\
\hline \multirow{3}{*}{$\begin{array}{l}\text { Deng et al. (2019) } \\
\text { Fan et al. (2018) } \\
\text { Grün et al. (2015) } \\
\text { Islam et al. (2014) } \\
\text { Zong et al. (2012) }\end{array}$} & \multirow{2}{*}{$\begin{array}{l}{[63]} \\
{[65]} \\
{[70]} \\
{[71]} \\
{[72]}\end{array}$} & $\begin{array}{l}\text { Trapnell et al. (2015) } \\
\text { Gawad et al. (2016) } \\
\text { Lappalainen et al. (2019) }\end{array}$ & $\begin{array}{l}{[75]} \\
{[83]} \\
{[84]}\end{array}$ & \multirow{2}{*}{$\begin{array}{l}\text { Stark et al. (2019) } \\
\text { Deng et al. }(2020) \\
\text { Erhard et al. }(2019) \\
\text { Hedlund et al. (2018) } \\
\text { Tang et al. (2009) }\end{array}$} & \multirow{2}{*}{$\begin{array}{l}{[66]} \\
{[67]} \\
{[68]} \\
{[69]} \\
{[73]}\end{array}$} & \multirow[t]{2}{*}{$\begin{array}{l}\text { Slavov et al. (2020) } \\
\text { Budnik et al. }(2018) \\
\text { Thul el at. (2017) }\end{array}$} & \multirow[t]{2}{*}{$\begin{array}{l}{[76]} \\
{[77]} \\
{[78]}\end{array}$} \\
\hline & & Single-cell epigenetics & & & & & \\
\hline & & $\begin{array}{l}\text { Ku et al. (2021) } \\
\text { Wang et al. (2019) }\end{array}$ & $\begin{array}{l}{[81]} \\
{[82]}\end{array}$ & & & & \\
\hline
\end{tabular}

Figure 2: Diagram of the relationship between the central dogma and DLBCL multi-omics studies. It includes the interrelationship between the central dogma (layer 1), multi-omics studies (layer 2), and recent DLBCL studies in single-cell multi-omics (layer 3). For example, the DNA changed in the biological cycle in the central dogma corresponds with the possible mutations, rearrangements and copy number variants in DLBCL at the genetic level. 
marrow, blood, or cerebrospinal fluid (CSF). Lymphoma tissue is definitely the best source for biomarker research. However, several limitations exist. Increasing evidences have indicated the great heterogeneity of DLBCL, even intratumoral in a single patient, making it unsuitable for reflecting the genetic landscape from a single-site biopsy. Moreover, it is inconvenient to repeat tumor biopsy to determine the genetic changes during treatment. With the advent of liquid biopsy, samples from blood or CSF could be used in this scenario, and could highly match the findings from tumor tissues.

Blood samples are least invasive, cost-effective, and easily accessible. Blood biopsy samples can be studied at multiple levels, such as the genome, transcriptome, proteome, and metabolome. They can be an important source to discover prognostic DLBCL biomarkers. Peripheral blood from DLBCL patients can be subjected to liquid biopsy to detect circulating tumor cells (CTCs) and circulating cell-free tumor DNA (ctDNA or cfDNA). CTCs and ctDNA are of great interest for early detection of disease and assessment of prognosis, but their sensitivity and specificity still need to be improved [96]. Rivas-Delgado $\mathrm{A}$ et al demonstrated in a population-based study that cfDNA could be an alternative source to assess the tumor burden and to show the tumor mutational profile and genetic classification, which have prognostic values and may guide future precision medicine in DLBCL [97]. Frank MJ et al [98] showed in a multi-center study that monitoring of ctDNA could help detect early relapse after CAR-T cell therapy in relapsed/refractory DLBCL. Moreover, several biomarkers easily detected in blood samples could also be used to predict prognosis of DLBCL. sFLC has been shown to be a predictor of poor prognosis in patients with DLBCL $[44,45]$. Bittenbring et al. found that EFS of DLBCL patients can be predicted based on serum Vitamin-D levels. The 3-year EFS was 59\% and 79\%, for DLBCL patients with $(\leq 8 \mathrm{ng} / \mathrm{ml})$ and without $(>8 \mathrm{ng} / \mathrm{ml})$ Vitamin-D deficiency, respectively. Vitamin-D levels were also found to affect the OS response to rituximab treatment; the 3-year OS rates were $70 \%$ and $82 \%$, respectively, for patients with and without Vitamin-D deficiency. In the multivariate analysis adjusted for IPI, the HR of EFS was $2.1(P=0.008)$, and the HR of OS was $1.9(P=0.040)$ and the prognosis of the two groups was significantly different. Treating for vitamin D deficiency (VDD) significantly increased rituximab-mediated cytotoxicity (RMCC) $(P<0.001)$, suggesting that VDD is a predictor of poor prognosis in elderly DLBCL patients [99]. In addition, Vaidya et al. proposed that absolute lymphocyte count (ALC) may also be a predictor of DLBCL prognosis in the era of $\mathrm{R}(\mathrm{X})$-CHOP [100]. In brief, given the non-invasive nature of liquid biopsy in peripheral blood and the rich resource of biomarkers in blood has not been fully exploited, it is believed that in future, blood will be a crucial source of sampling in the search for prognostic biomarkers in DLBCL.

Central nervous system (CNS) is a major site of DLBCL recurrence in many patients. CSF composition is altered in response to cancer. Lumbar puncture and CSF testing of patients can reveal that certain biomarkers have predictive significance for the prognosis of DLBCL patients. IL-10/IL-6 ratio, which is one of the indicators of poor prognosis of DLBCL, increased in the CSF of primary CNS lymphoma (PCNSL) patients. Kishimoto et al. also determined that TIM-1 is related to high expression of IL-10 [101]. Muñiz et al. used FCM to evaluate B cell-related markers in the CSF of patients with CNS lymphoma and suggested that higher levels of soluble CD19 (sCD19) were associated with CNS relapse rates in DLBCL patients, which are predictors of poor prognosis in DLBCL patients [102]. Alvarez et al. found that elevated lactate dehydrogenase (LDH) levels were associated with an increased risk of CNS relapse [103]. Cairo et al. also showed that elevated LDH levels were associated with an increased risk of CNS recurrence in adolescent (15-21 years) DLBCL patients [104]. Moreover, ctDNA in the CSF plays an important role in the application of liquid biopsy in patients with CNS cancers [105]. In the clinic, the diagnosis of CNS involvement in DLBCL patients is based on several clinical risk factors, including IPI, $\mathrm{LDH}$, and the number of extra-nodal involvement (testis/adrenal gland/kidney) [106]. A six-risk factor CNS-IPI model (five IPI factors with renal/adrenal gland involvement) developed by the German group for CNS diagnosis has been used in the clinic [107]. In January 2021, Wang et al. published a new progress in the study of CSF-ctDNA in DLBCL patients. They found that increased free DNA (cfDNA) concentrations in the CSF of DLBCL patients correlated with high CNS-IPI. These findings underscore the significance of CSF-cfDNA in the detecting CNS tumors in DLBCL patients [108]. Thus, CSF-cfDNA may be a putative prognostic biomarker in DLBCL patients.

\section{Conclusions}

Predicting and determining the prognosis of patients with DLBCL is a long and complex research process. In the era of precision therapy, molecular typing of DLBCL using new technologies further enhances our understanding of the prognostic factors and biological behavior of DLBCL tumors. New molecular typing will play an important role in 
predicting the prognosis of DLBCL patients and will help to develop individualized treatment plans for patients and improve the cure and survival rates of DLBCL patients. In recent decades, research on DLBCL has advanced at multiple levels. The analysis of histological information and other databases has led to the discovery of multiple biomarkers that are predictive of therapeutic outcomes. Interestingly, single-cell histological studies of DLBCL are progressing rapidly, but have not yet been truly applied in the clinic. There is an urgent need to develop single-cell technologies in the future to promote novel biomarkers based on single-cell histological studies. Large scale clinical studies examining the presence of mutants, the impact of different biomarkers on the prognosis of treatment, the intricate network of signaling pathways within and between tumor cells, and whether traditional drugs and new targeted drugs can obtain reliable and clinically guided prognostic results based on new molecular DLBCL subtyping need to be conducted. In addition, studies on new research methods (aimed at reducing operational difficulty, cost, and time) with adequate sample size have become necessary for DLBCL research.

In summary, despite the many challenges of systems biology research, the integration of multi-omics studies continues to be a great boost to the identification of novel biomarkers of DLBCL.

\section{Abbreviations}

2D-LC: two-dimensional chromatography; $\mathrm{ABC}$ : activated B cells; ALC: absolute lymphocyte count; B2M: $\beta 2$-microglobulin; BATF: basic leucine zipper ATF-like transcription factor; CAR: chimeric antigen receptor; $\mathrm{CI}$ : confidence interval; $\mathrm{COO}$ : cell of origin; CR: complete remission; CSF: cerebrospinal fluid; CNS: Central nervous system; CT: computed tomography; CTCs: circulating tumor cells; ctDNA: Circulating tumor DNA; DHITsig: double-hit signature; DLBCL: Diffuse large B cell lymphoma; EFS: event free survival; EMR: early molecular response; EMR: early molecular response; FOXP1: forkhead box protein 1; GAPDH: glyceraldehyde-3phosphate dehydrogenase; GEP: gene expression profile; GCB: germinal center B cells; HRs: hazard ratios; IFNy: interferon gamma; IHC: immunohistochemistry; IPI: International Prognostic Index; LME: lymphoma microenvironment; LC-MS/MS: two-dimensional liquid chromatography/tandem mass spectrometry; lncRNAs: long non-coding RNAs; m6A: N6-methyladenosine; miRNAs: microRNAs; MLL2: mixed-lineage leukemia 2; MMR: major molecular response; NEC: not elsewhere classified; NGS: next-generation sequencing technology;
non-GCB: non-germinal center B cell-like; NPV: negative predictive value; NK cells: natural killer cells; OS: overall survival; Oxphos: oxidative phosphorylation; PCNSL: primary CNS lymphoma; PD: progressive disease; PD-1: programmed death receptor 1; PD-L1: programmed death receptor-ligand 1; PET-CT: positron emission computed tomography; PFS: progression free survival; PPV: positive predictive value; PR: partial remission; PS: patient survival; PY-STAT3: phosphotyrosine STAT3; qPCR: real-time quantitative PCR; R-CHOP: rituximab, cyclophosphamide, adriamycin, vincristine, and prednisone; RNA-seq: RNA sequencing; SCNAs: somatic copy number alterations; scRNA-seq: single-cell RNA sequencing; sFLC: serum free light chain; SNP: single nucleotide polymorphism; SUV: standard uptake value; SVs: structural variants; TMA: tissue microarray; TME: tumor microenvironment; TMTV: total metabolic tumor volume; VDD: vitamin D deficiency.

\section{Acknowledgements}

\section{Funding}

This work was financially supported through grants from the National Natural Science Foundation of China (NO. 81873450, NO. 82170181, NO. 81770170 and NO. 81970147), and Beijing Hospitals Authority Youth Programme (code: QML20200201) to Liang Wang.

\section{Author Contributions}

XJL, XYS and JLW collected the related papers and wrote the manuscript. DL and BYL participated in the design of the review. HSZ and LW initiated the study and revised and finalized the manuscript. All authors read and approved the final manuscript.

\section{Competing Interests}

The authors have declared that no competing interest exists.

\section{References}

1. Chapuy B, Stewart C, Dunford AJ, Kim J, Kamburov A, Redd RA, et al. Molecular subtypes of diffuse large B cell lymphoma are associated with distinct pathogenic mechanisms and outcomes. Nat Med. 2018; 24: $679-90$

2. Flowers CR, Sinha R, Vose JM. Improving outcomes for patients with diffuse large B-cell lymphoma. CA Cancer J Clin. 2010; 60: 393-408.

3. Matthews JM, Bhatt S, Patricelli MP, Nomanbhoy TK, Jiang $X$, Natkunam Y, et al. Pathophysiological significance and therapeutic targeting of germinal center kinase in diffuse large B-cell lymphoma. Blood. 2016; 128: 239-48.

4. Miao Y, Medeiros LJ, Li Y, Li J, Young KH. Genetic alterations and their clinical implications in DLBCL. Nat Rev Clin Oncol. 2019; 16: 634-52.

5. Alizadeh AA, Eisen MB, Davis RE, Ma C, Lossos IS, Rosenwald A, et al. Distinct types of diffuse large B-cell lymphoma identified by gene expression profiling. Nature. 2000; 403: 503-11.

6. Rosenwald A, Wright G, Chan WC, Connors JM, Campo E, Fisher RI, et al. The use of molecular profiling to predict survival after chemotherapy for diffuse large-B-cell lymphoma. N Engl J Med. 2002; 346: 1937-47. 
7. Hans CP, Weisenburger DD, Greiner TC, Gascoyne RD, Delabie J, Ott G, et al. Confirmation of the molecular classification of diffuse large B-cell lymphoma by immunohistochemistry using a tissue microarray. Blood. 2004; 103: 275-82

8. Choi WWL, Weisenburger DD, Greiner TC, Piris MA, Banham AH, Delabie J, et al. A new immunostain algorithm classifies diffuse large B-cell lymphoma into molecular subtypes with high accuracy. Clin Cancer Res. 2009; 15: 5494-502.

9. Schmitz R, Wright GW, Huang DW, Johnson CA, Phelan JD, Wang JQ, et al. Genetics and Pathogenesis of Diffuse Large B-Cell Lymphoma. N Engl J Med. 2018; 378: 1396-407.

10. Lionakis MS, Dunleavy K, Roschewski M, Widemann BC, Butman JA, Schmitz R, et al. Inhibition of B Cell Receptor Signaling by Ibrutinib in Primary CNS Lymphoma. Cancer Cell. 2017; 31.

11. Lacy SE, Barrans SL, Beer PA, Painter D, Smith AG, Roman E, et al. Targeted sequencing in DLBCL, molecular subtypes, and outcomes: a Haematological Malignancy Research Network report. Blood. 2020; 135: 1759-71.

12. Wright GW, Huang DW, Phelan JD, Coulibaly ZA, Roulland S, Young RM, et al. A Probabilistic Classification Tool for Genetic Subtypes of Diffuse Large B Cell Lymphoma with Therapeutic Implications. Cancer Cell. 2020; 37: 551-68.e14

13. Zhang $M, X u$ P, Wang L, Cheng S, Zhao W. GENETIC SUBTYPE GUIDED RITUXIMAB-BASED IMMUNOCHEMOTHERAPY IMPROVES OUTCOME IN NEWLY DIAGNOSED DIFFUSE LARGE B-CELL LYMPHOMA: FIRST REPORT OF A RANDOMIZED PHASE 2 STUDY. 2021; 39.

14. Kotlov N, Bagaev A, Revuelta MV, Phillip JM, Cacciapuoti MT, Antysheva Z, et al. Clinical and Biological Subtypes of B-cell Lymphoma Revealed by Microenvironmental Signatures. Cancer Discov. 2021; 11: 1468-89.

15. Lynch RC, Gratzinger D, Advani RH. Clinical Impact of the 2016 Update to the WHO Lymphoma Classification. Curr Treat Options Oncol. 2017; 18: 45 .

16. Savage KJ, Slack GW, Mottok A, Sehn LH, Villa D, Kansara R, et al. Impact of dual expression of MYC and BCL2 by immunohistochemistry on the risk of CNS relapse in DLBCL. Blood. 2016; 127: 2182-8.

17. Horn H, Ziepert M, Becher C, Barth TF, Bernd HW, Feller AC, et al. MYC status in concert with BCL2 and BCL6 expression predicts outcome in diffuse large B-cell lymphoma. Blood. 2013; 121: 2253-63.

18. Dunleavy K, Fanale MA, Abramson JS, Noy A, Caimi PF, Pittaluga S, et al. Dose-adjusted EPOCH-R (etoposide, prednisone, vincristine, cyclophosphamide, doxorubicin, and rituximab) in untreated aggressive diffuse large B-cell lymphoma with MYC rearrangement: a prospective, multicentre, single-arm phase 2 study. Lancet Haematol. 2018; 5: e609-e17.

19. Staiger AM, Ziepert M, Horn H, Scott DW, Barth TFE, Bernd HW, et al. Clinical Impact of the Cell-of-Origin Classification and the MYC/ BCL2 Dual Expresser Status in Diffuse Large B-Cell Lymphoma Treated Within Prospective Clinical Trials of the German High-Grade Non-Hodgkin's Lymphoma Study Group. J Clin Oncol. 2017; 35: 2515-26.

20. Gupta M, Maurer MJ, Wellik LE, Law ME, Han JJ, Ozsan N, et al. Expression of Myc, but not pSTAT3, is an adverse prognostic factor for diffuse large B-cell lymphoma treated with epratuzumab/R-CHOP. Blood. 2012; 120: 4400-6.

21. Huang X, Meng B, Iqbal J, Ding BB, Perry AM, Cao W, et al. Activation of the STAT3 signaling pathway is associated with poor survival in diffuse large B-cell lymphoma treated with R-CHOP. J Clin Oncol. 2013; 31: 4520-8.

22. Ghesquieres H, Slager SL, Jardin F, Veron AS, Asmann YW, Maurer MJ, et al. Genome-Wide Association Study of Event-Free Survival in Diffuse Large B-Cell Lymphoma Treated With Immunochemotherapy. J Clin Oncol. 2015; 33: 3930-7.

23. Pasqualucci L, Trifonov V, Fabbri G, Ma J, Rossi D, Chiarenza A, et al. Analysis of the coding genome of diffuse large B-cell lymphoma. Nat Genet. 2011; 43: 830-7.

24. Reddy A, Zhang J, Davis NS, Moffitt AB, Love CL, Waldrop A, et al. Genetic and Functional Drivers of Diffuse Large B Cell Lymphoma. Cell. 2017; 171: 481-94.e15.

25. Bettegowda C, Sausen M, Leary RJ, Kinde I, Wang Y, Agrawal N, et al. Detection of circulating tumor DNA in early- and late-stage human malignancies. Sci Transl Med. 2014; 6: 224ra24.

26. Diehl F, Schmidt K, Choti MA, Romans K, Goodman S, Li M, et al. Circulating mutant DNA to assess tumor dynamics. Nat Med. 2008; 14 : 985-90.

27. Alig S, Macaulay CW, Kurtz DM, Dührsen U, Hüttmann A, Schmitz C, et al. Short Diagnosis-to-Treatment Interval Is Associated With Higher Circulating Tumor DNA Levels in Diffuse Large B-Cell Lymphoma. J Clin Oncol. 2021: Jco2002573.
28. Sidaway P. ctDNA predicts outcomes in DLBCL. Nat Rev Clin Oncol. 2018; 15: 655.

29. Roschewski M, Dunleavy K, Pittaluga S, Moorhead M, Pepin F, Kong K, et al. Circulating tumour DNA and CT monitoring in patients with untreated diffuse large B-cell lymphoma: a correlative biomarker study. Lancet Oncol. 2015; 16: 541-9.

30. Ennishi D, Jiang A, Boyle M, Collinge B, Grande BM, Ben-Neriah S, et al. Double-Hit Gene Expression Signature Defines a Distinct Subgroup of Germinal Center B-Cell-Like Diffuse Large B-Cell Lymphoma. J Clin Oncol. 2019; 37: 190-201.

31. Ciavarella S, Vegliante MC, Fabbri M, De Summa S, Melle F, Motta G, et al. Dissection of DLBCL microenvironment provides a gene expression-based predictor of survival applicable to formalin-fixed paraffin-embedded tissue. Ann Oncol. 2018; 29: 2363-70.

32. Sun $\mathrm{R}$, Zheng Z, Wang $\mathrm{L}$, Cheng $\mathrm{S}$, Shi Q, Qu B, et al. A novel prognostic model based on four circulating miRNA in diffuse large B-cell lymphoma: implications for the roles of MDSC and Th17 cells in lymphoma progression Mol Oncol. 2021; 15: 246-61.

33. Bouvy C, Wannez A, George F, Graux C, Chatelain C, Dogné JM. Circulating MicroRNAs as Biomarkers in Diffuse Large B-cell Lymphoma: A Pilot Prospective Longitudinal Clinical Study. Biomark Cancer. 2018; 10: 1179299x18781095.

34. Zhou M, Zhao H, Xu W, Bao S, Cheng L, Sun J. Discovery and validation of immune-associated long non-coding RNA biomarkers associated with clinically molecular subtype and prognosis in diffuse large B cell lymphoma. Mol Cancer. 2017; 16: 16.

35. Bachy E, Coiffier B. Anti-PD1 antibody: a new approach to treatment of lymphomas. Lancet Oncol. 2014; 15: 7-8.

36. Ansell SM, Lesokhin AM, Borrello I, Halwani A, Scott EC, Gutierrez M, et al. PD-1 blockade with nivolumab in relapsed or refractory Hodgkin's lymphoma. N Engl J Med. 2015; 372: 311-9.

37. Kiyasu J, Miyoshi H, Hirata A, Arakawa F, Ichikawa A, Niino D, et al. Expression of programmed cell death ligand 1 is associated with poor overall survival in patients with diffuse large B-cell lymphoma. Blood. 2015; 126: 2193-201

38. Flori M, Schmid CA, Sumrall ET, Tzankov A, Law CW, Robinson MD, et al. The hematopoietic oncoprotein FOXP1 promotes tumor cell survival in diffuse large B-cell lymphoma by repressing S1PR2 signaling. Blood. 2016; 127: 1438-48.

39. Green JA, Suzuki K, Cho B, Willison LD, Palmer D, Allen CD, et al. The sphingosine 1-phosphate receptor $\mathrm{S}_{1} \mathrm{P}_{2}$ maintains the homeostasis of germinal center B cells and promotes niche confinement. Nat Immunol. 2011; 12: 672-80.

40. Niitsu N, Okamoto M, Tamaru JI, Yoshino T, Nakamura N, Nakamura S, et al. Clinicopathologic characteristics and treatment outcome of the addition of rituximab to chemotherapy for CD5-positive in comparison with CD5-negative diffuse large B-cell lymphoma. Ann Oncol. 2010; 21: 2069-74.

41. Xu-Monette ZY, Li L, Byrd JC, Jabbar KJ, Manyam GC, Maria de Winde $\mathrm{C}$, et al. Assessment of CD37 B-cell antigen and cell of origin significantly improves risk prediction in diffuse large B-cell lymphoma. Blood. 2016; 128: 3083-100.

42. Meriranta L, Pasanen A, Cervera A, Holte H, Jr., Lehtonen R, Hautaniemi S, et al. Low Expression and Somatic Mutations of the KLHL6 Gene Predict Poor Survival in Patients with Activated B-Cell like Diffuse Large B-Cell Lymphoma. Blood. 2016; 128: 2926-.

43. Bradwell AR, Carr-Smith HD, Mead GP, Tang LX, Showell PJ, Drayson MT, et al. Highly sensitive, automated immunoassay for immunoglobulin free light chains in serum and urine. Clin Chem. 2001; 47: 673-80.

44. Maurer MJ, Micallef IN, Cerhan JR, Katzmann JA, Link BK, Colgan JP, et al. Elevated serum free light chains are associated with event-free and overall survival in two independent cohorts of patients with diffuse large B-cell lymphoma. J Clin Oncol. 2011; 29: 1620-6.

45. Witzig TE, Maurer MJ, Stenson MJ, Allmer C, Macon W, Link B, et al. Elevated serum monoclonal and polyclonal free light chains and interferon inducible protein-10 predicts inferior prognosis in untreated diffuse large B-cell lymphoma. Am J Hematol. 2014; 89: 417-22.

46. Uzun $\mathrm{Y}, \mathrm{Wu} \mathrm{H}$, Tan $\mathrm{K}$. Predictive modeling of single-cell DNA methylome data enhances integration with transcriptome data. Genome Res. 2021; 31: 101-9.

47. Armand EJ, Li J, Xie F, Luo C, Mukamel EA. Single-Cell Sequencing of Brain Cell Transcriptomes and Epigenomes. Neuron. 2021; 109: 11-26.

48. Friedman N, Rando OJ. Epigenomics and the structure of the living genome. Genome Res. 2015; 25: 1482-90.

49. Karemaker ID, Vermeulen M. Single-Cell DNA Methylation Profiling: Technologies and Biological Applications. Trends Biotechnol. 2018; 36: 952-65. 
50. Pan H, Jiang Y, Boi M, Tabbò F, Redmond D, Nie K, et al. Epigenomic evolution in diffuse large B-cell lymphomas. Nat Commun. 2015; 6: 6921.

51. Teater M, Dominguez PM, Redmond D, Chen Z, Ennishi D, Scott DW, et al. AICDA drives epigenetic heterogeneity and accelerates germinal center-derived lymphomagenesis. Nat Commun. 2018; 9: 222

52. Jiang $X$, Liu B, Nie Z, Duan L, Xiong Q, Jin Z, et al. The role of m6A modification in the biological functions and diseases. Signal Transduct Target Ther. 2021; 6: 74 .

53. Han H, Fan G, Song S, Jiang Y, Qian C, Zhang W, et al. piRNA-30473 contributes to tumorigenesis and poor prognosis by regulating $\mathrm{m} 6 \mathrm{~A}$ RNA methylation in DLBCL. Blood. 2021; 137: 1603-14.

54. Guan XW, Wang HQ, Ban WW, Chang Z, Chen HZ, Jia L, et al. Novel HDAC inhibitor Chidamide synergizes with Rituximab to inhibit diffuse large B-cell lymphoma tumour growth by upregulating CD20. Cell Death Dis. 2020; 11: 20

55. Monti S, Savage KJ, Kutok JL, Feuerhake F, Kurtin P, Mihm M, et al Molecular profiling of diffuse large B-cell lymphoma identifies robust subtypes including one characterized by host inflammatory response. Blood. 2005; 105: 1851-61.

56. Caro P, Kishan AU, Norberg E, Stanley IA, Chapuy B, Ficarro SB, et al. Metabolic signatures uncover distinct targets in molecular subsets of diffuse large B cell lymphoma. Cancer Cell. 2012; 22: 547-60.

57. Ceriani L, Martelli M, Zinzani PL, Ferreri AJ, Botto B, Stelitano C, et al. Utility of baseline 18FDG-PET/CT functional parameters in defining prognosis of primary mediastinal (thymic) large B-cell lymphoma. Blood. 2015; 126: 950-6.

58. Koppenol WH, Bounds PL, Dang CV. Otto Warburg's contributions to current concepts of cancer metabolism. Nat Rev Cancer. 2011; 11: 325-37.

59. Chiche J, Reverso-Meinietti J, Mouchotte A, Rubio-Patiño C, Mhaidly R, Villa E, et al. GAPDH Expression Predicts the Response to R-CHOP, the Tumor Metabolic Status, and the Response of DLBCL Patients to Metabolic Inhibitors. Cell Metab. 2019; 29: 1243-57.e10.

60. Feist M, Schwarzfischer P, Heinrich P, Sun X, Kemper J, von Bonin F, et al. Cooperative STAT/NF-kB signaling regulates lymphoma metabolic reprogramming and aberrant GOT2 expression. Nat Commun. 2018; 9: 1514

61. Tome ME, Johnson DB, Rimsza LM, Roberts RA, Grogan TM, Miller TP, et al. A redox signature score identifies diffuse large B-cell lymphoma patients with a poor prognosis. Blood. 2005; 106: 3594-601.

62. Kobayashi T, Lam PY, Jiang H, Bednarska K, Gloury R, Murigneux V, et al. Increased lipid metabolism impairs NK cell function and mediates adaptation to the lymphoma environment. Blood. 2020; 136: 3004-17.

63. Deng Y, Finck A, Fan R. Single-Cell Omics Analyses Enabled by Microchip Technologies. Annu Rev Biomed Eng. 2019; 21: 365-93.

64. Suvà ML, Tirosh I. Single-Cell RNA Sequencing in Cancer: Lessons Learned and Emerging Challenges. Mol Cell. 2019; 75: 7-12.

65. Fan J, Lee HO, Lee S, Ryu DE, Lee S, Xue C, et al. Linking transcriptional and genetic tumor heterogeneity through allele analysis of single-cell RNA-seq data. Genome Res. 2018; 28: 1217-27.

66. Stark R, Grzelak M, Hadfield J. RNA sequencing: the teenage years. Nat Rev Genet. 2019; 20: 631-56.

67. Deng Q, Han G, Puebla-Osorio N, Ma MCJ, Strati P, Chasen B, et al. Characteristics of anti-CD19 CAR T cell infusion products associated with efficacy and toxicity in patients with large B cell lymphomas. Nat Med. 2020; 26: 1878-87.

68. Erhard F, Baptista MAP, Krammer T, Hennig T, Lange M, Arampatzi P, et al. scSLAM-seq reveals core features of transcription dynamics in single cells. Nature. 2019; 571: 419-23.

69. Hedlund E, Deng Q. Single-cell RNA sequencing: Technical advancements and biological applications. Mol Aspects Med. 2018; 59: 36-46.

70. Grün D, van Oudenaarden A. Design and Analysis of Single-Cell Sequencing Experiments. Cell. 2015; 163: 799-810.

71. Islam S, Zeisel A, Joost S, La Manno G, Zajac P, Kasper M, et al. Quantitative single-cell RNA-seq with unique molecular identifiers. Nat Methods. 2014; 11: 163-6.

72. Zong C, Lu S, Chapman AR, Xie XS. Genome-wide detection of single-nucleotide and copy-number variations of a single human cell. Science. 2012; 338: 1622-6.

73. Tang F, Barbacioru C, Wang Y, Nordman E, Lee C, Xu N, et al. mRNA-Seq whole-transcriptome analysis of a single cell. Nat Methods. 2009; 6: 377-82.

74. Zhang M, Zou Y, Xu X, Zhang X, Gao M, Song J, et al. Highly parallel and efficient single cell mRNA sequencing with paired picoliter chambers. Nat Commun. 2020; 11: 2118.

75. Trapnell C. Defining cell types and states with single-cell genomics. Genome Res. 2015; 25: 1491-8.

76. Slavov N. Unpicking the proteome in single cells. Science. 2020; 367: 512-3.
77. Budnik B, Levy E, Harmange G, Slavov N. SCoPE-MS: mass spectrometry of single mammalian cells quantifies proteome heterogeneity during cell differentiation. Genome Biol. 2018; 19: 161.

78. Thul PJ, Åkesson L, Wiking M, Mahdessian D, Geladaki A, Ait Blal H, et al. A subcellular map of the human proteome. Science. 2017; 356.

79. Bendall SC, Simonds EF, Qiu P, Amir el AD, Krutzik PO, Finck R, et al. Single-cell mass cytometry of differential immune and drug responses across a human hematopoietic continuum. Science. 2011; 332: 687-96.

80. Hughes AJ, Spelke DP, Xu Z, Kang CC, Schaffer DV, Herr AE. Single-cell western blotting. Nat Methods. 2014; 11: 749-55.

81. Ku WL, Pan L, Cao Y, Gao W, Zhao K. Profiling single-cell histone modifications using indexing chromatin immunocleavage sequencing. Genome Res. 2021.

82. Wang Q, Xiong H, Ai S, Yu X, Liu Y, Zhang J, et al. CoBATCH for High-Throughput Single-Cell Epigenomic Profiling. Mol Cell. 2019; 76: 206-16.e7.

83. Gawad C, Koh W, Quake SR. Single-cell genome sequencing: current state of the science. Nat Rev Genet. 2016; 17: 175-88.

84. Lappalainen T, Scott AJ, Brandt M, Hall IM. Genomic Analysis in the Age of Human Genome Sequencing. Cell. 2019; 177: 70-84.

85. Doerr A. Single-cell proteomics. Nat Methods. 2019; 16: 20.

86. Shapiro E, Biezuner T, Linnarsson S. Single-cell sequencing-based technologies will revolutionize whole-organism science. Nat Rev Genet. 2013; 14: 618-30.

87. Rodriguez H, Zenklusen JC, Staudt LM, Doroshow JH, Lowy DR. The next horizon in precision oncology: Proteogenomics to inform cancer diagnosis and treatment. Cell. 2021; 184: 1661-70.

88. Cheson BD, Fisher RI, Barrington SF, Cavalli F, Schwartz LH, Zucca E, et al. Recommendations for initial evaluation, staging, and response assessment of Hodgkin and non-Hodgkin lymphoma: the Lugano classification. J Clin Oncol. 2014; 32: 3059-68.

89. Barrington SF, Mikhaeel NG, Kostakoglu L, Meignan M, Hutchings M, Müeller SP, et al. Role of imaging in the staging and response assessment of lymphoma: consensus of the International Conference on Malignant Lymphomas Imaging Working Group. J Clin Oncol. 2014; 32: 3048-58.

90. Xie W, Liu MK, Jiang XF, Gao XD, Li B, Wang L, et al. Improved prediction of chemoresistance in patients with diffuse large B-cell lymphoma through a new interim positron emission tomography-computed tomography evaluation model. Acta Oncol. 2021: $1-9$.

91. Zhou H, Xu-Monette ZY, Xiao L, Strati P, Hagemeister FB, He Y, et al. Prognostic factors, therapeutic approaches, and distinct immunobiologic features in patients with primary mediastinal large B-cell lymphoma on long-term follow-up. Blood Cancer J. 2020; 10: 49.

92. Mamot C, Klingbiel D, Hitz F, Renner C, Pabst T, Driessen C, et al. Final Results of a Prospective Evaluation of the Predictive Value of Interim Positron Emission Tomography in Patients With Diffuse Large B-Cell Lymphoma Treated With R-CHOP-14 (SAKK 38/07). J Clin Oncol. 2015; 33: 2523-9.

93. Held G, Murawski N, Ziepert M, Fleckenstein J, Pöschel V, Zwick C, et al. Role of radiotherapy to bulky disease in elderly patients with aggressive B-cell lymphoma. J Clin Oncol. 2014; 32: 1112-8.

94. Vercellino L, Cottereau AS, Casasnovas O, Tilly H, Feugier P, Chartier L, et al. High total metabolic tumor volume at baseline predicts survival independent of response to therapy. Blood. 2020; 135: 1396-405.

95. Lue KH, Wu YF, Lin HH, Hsieh TC, Liu SH, Chan SC, et al. Prognostic Value of Baseline Radiomic Features of (18)F-FDG PET in Patients with Diffuse Large B-Cell Lymphoma. Diagnostics (Basel). 2020; 11.

96. Alix-Panabières C, Pantel K. Clinical Applications of Circulating Tumor Cells and Circulating Tumor DNA as Liquid Biopsy. Cancer Discov. 2016; 6: 479-91.

97. Rivas-Delgado A, Nadeu F, Enjuanes A, Casanueva-Eliceiry S, Mozas P, Magnano L, et al. Mutational Landscape and Tumor Burden Assessed by Cell-free DNA in Diffuse Large B-Cell Lymphoma in a Population-Based Study. Clin Cancer Res. 2021; 27: 513-21.

98. Frank MJ, Hossain NM, Bukhari A, Dean E, Spiegel JY, Claire GK, et al. Monitoring of Circulating Tumor DNA Improves Early Relapse Detection After Axicabtagene Ciloleucel Infusion in Large B-Cell Lymphoma: Results of a Prospective Multi-Institutional Trial. J Clin Oncol. 2021; 39: 3034-43.

99. Bittenbring JT, Neumann F, Altmann B, Achenbach M, Reichrath J, Ziepert $\mathrm{M}$, et al. Vitamin D deficiency impairs rituximab-mediated cellular cytotoxicity and outcome of patients with diffuse large B-cell lymphoma treated with but not without rituximab. J Clin Oncol. 2014; 32: 3242-8.

100. Vaidya R, Witzig TE. Prognostic factors for diffuse large B-cell lymphoma in the R(X)CHOP era. Ann Oncol. 2014; 25: 2124-33. 
101. Kishimoto W, Nishikori M, Arima H, Miyoshi H, Sasaki Y, Kitawaki T, et al. Expression of Tim-1 in primary CNS lymphoma. Cancer Med. 2016; 5: 3235-45.

102. Muñiz C, Martín-Martín L, López A, Sánchez-González B, Salar A, Almeida J, et al. Contribution of cerebrospinal fluid SCD19 levels to the detection of CNS lymphoma and its impact on disease outcome. Blood. 2014; 123: 1864-9.

103. Alvarez R, Dupuis J, Plonquet A, Christov C, Copie-Bergman C, Hemery $\mathrm{F}$, et al. Clinical relevance of flow cytometric immunophenotyping of the cerebrospinal fluid in patients with diffuse large B-cell lymphoma. Ann Oncol. 2012; 23: 1274-9.

104. Cairo MS, Sposto R, Gerrard M, Auperin A, Goldman SC, Harrison L, et al. Advanced stage, increased lactate dehydrogenase, and primary site, but not adolescent age ( $\geq 15$ years), are associated with an increased risk of treatment failure in children and adolescents with mature B-cell non-Hodgkin's lymphoma: results of the FAB LMB 96 study. J Clin Oncol. 2012; 30: 387-93.

105. Seoane J, De Mattos-Arruda L, Le Rhun E, Bardelli A, Weller M. Cerebrospinal fluid cell-free tumour DNA as a liquid biopsy for primary brain tumours and central nervous system metastases. Ann Oncol. 2019; 30: 211-8.

106. Kridel R, Dietrich PY. Prevention of CNS relapse in diffuse large B-cell lymphoma. Lancet Oncol. 2011; 12: 1258-66.

107. Schmitz N, Zeynalova S, Nickelsen M, Kansara R, Villa D, Sehn LH, et al. CNS International Prognostic Index: A Risk Model for CNS Relapse in Patients With Diffuse Large B-Cell Lymphoma Treated With R-CHOP. J Clin Oncol. 2016; 34: 3150-6.

108. Wang X, Gao Y, Shan C, Lai M, He H, Bai B, et al. Association of circulating tumor DNA from the cerebrospinal fluid with high-risk CNS involvement in patients with diffuse large B-cell lymphoma. Clin Transl Med. 2021; 11: e236. 